\title{
EFFECTIVENESS OF CARBAMIDE PEROXIDE AND SODIUM PERBORATE IN NON-VITAL DISCOLORED TEETH
}

\author{
Marcia Carneiro VALERA ${ }^{1}$, Carlos Henrique Ribeiro CAMARGO², Cláudio Antonio Talge CARVALHO ${ }^{3}$, \\ Luciane Dias de OLIVEIRA ${ }^{4}$, Samira Esteves Afonso CAMARGO ${ }^{5}$, Cristiana Martins RODRIGUES ${ }^{6}$
}

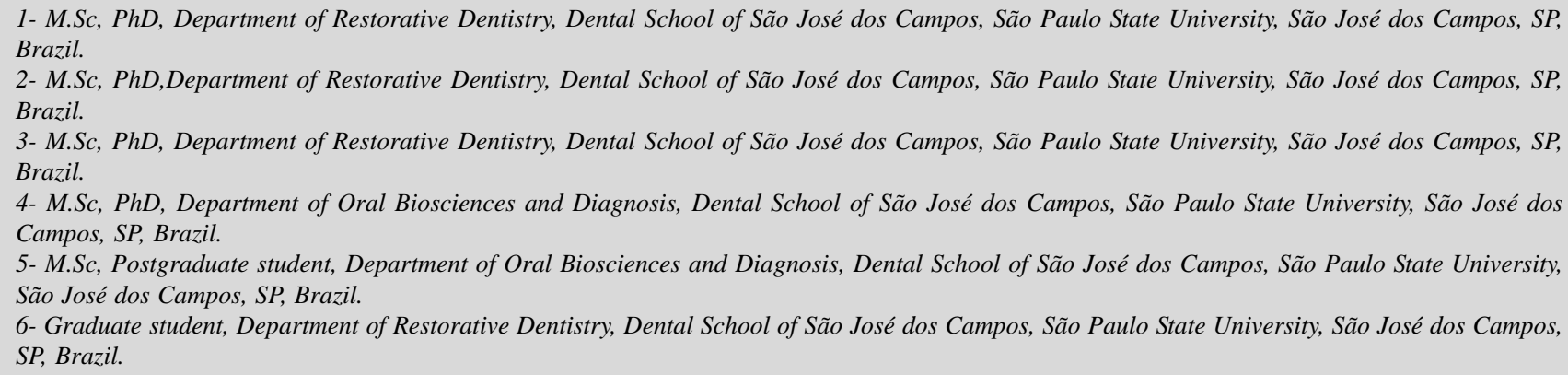

4- M.Sc, PhD, Department of Oral Biosciences and Diagnosis, Dental School of São José dos Campos, São Paulo State University, São José dos Campos, SP, Brazil.

5- M.Sc, Postgraduate student, Department of Oral Biosciences and Diagnosis, Dental School of São José dos Campos, São Paulo State University, São José dos Campos, SP, Brazil.

6- Graduate student, Department of Restorative Dentistry, Dental School of São José dos Campos, São Paulo State University, São José dos Campos, SP, Brazil.

Corresponding address: Luciane Dias de Oliveira - Departamento: Biociências e Diagnóstico Bucal - Av. Francisco José Longo, 777 - Jd. São Dimas 12245-000 - São José dos Campos, SP - Brasil - e-mail: Luciane@fosjc.unesp.br

Received: May 9, 2008 - Modification: August 31, 2008 - Accepted: October 7, 2008

\begin{abstract}
$O$

bjective: To evaluate the efficacy of $16 \%$ carbamide peroxide gel (CP16\%), tetrahydrate sodium perborate (SP) and mixture $(\mathrm{CP} 16 \%+\mathrm{SP})$, in walking bleaching of non-vital discolored teeth. Materials and methods: Sixty single-rooted human premolars with intact crowns were used and initial color was assessed using Vita shade guide and standardized photos. The teeth were stained using rabbit fresh blood for 18 days and photos of discolored teeth and color evaluation were performed. The teeth were divided into 4 groups ( $\mathrm{n}=15$ ), according to bleaching agent used: G1) CP16\% gel; G2) CP16\% gel + SP; G3) SP + distilled water; G4: control. The bleaching agents were replaced twice at 7-day intervals for 21 days. All teeth were evaluated by two endodontists at days 0,7 , 14 and 21 and the color changes were assessed using Vita shade guide and standardized photos. The results were analyzed by Kruskal-Wallis and Dunn's tests $(\mathrm{p}=0.05)$. Results: The experimental groups presented statistically similar bleaching results ( $>0.05)$ at the end of 7, 14 and 21 days. These groups presented significantly higher bleaching efficacy than control group (G4) (p<0.05). The mixture CP16\% + SP promoted return of original color in $100 \%$ of teeth at the end of 21 days. Conclusion: It was concluded that three bleaching agents were effective in bleaching of stained teeth with blood products, especially at the end of 21 days.
\end{abstract}

Key words: Tetrahydrate sodium perborate. Carbamide peroxide. Non-vital discolored teeth. Bleaching.

\section{INTRODUCTION}

Tooth bleaching presents great importance in dental esthetics. In discolored non-vital teeth, the intracoronal bleaching procedure is widely used because it is efficient, relatively simple, presents low cost and preserves the dental hard tissue compared to the prosthetic treatment ${ }^{2}$.

Discoloration of non-vital teeth can be due to extrinsic or intrinsic factors ${ }^{24}$. The main intrinsic factors are pulp hemorrhage, decomposition of pulp, bacteria and its products, tetracycline, pulp necrosis, intracanal medicaments, some endodontic filling materials, metallic restorations, among others ${ }^{3,24}$. With the dissemination of blood components into the dentinal tubules caused by pulp extirpation or traumatically induced internal pulp bleeding ${ }^{8}$, blood vessels are broken, promoting of blood overflow into the pulp chamber. Blood degradation products such as haemosiderin, haemin, haematin and haematoidin release iron during haemolysis. The iron can be converted to black ferric sulphide with hydrogen sulphide produced by bacteria, which causes a grey staining of tooth. Besides the blood degradation, degrading proteins of necrotic pulp tissue may also cause discoloration ${ }^{2,8,21,25,29}$.

Several bleaching techniques and materials have been proposed in order to obtain satisfactory bleaching and no damages in dental hard tissues and periodontal ligament. 
The intracoronal bleaching agents most commonly used are hydrogen peroxide, carbamide peroxide and sodium perborate ${ }^{17,28,30}$. Hydrogen peroxide presents great effectiveness to remove stains in enamel and dentin of vital and non-vital teeth. Carbamide peroxide, which releases hydrogen peroxide and urea, was introduced in 1989 by Haywood and Heymann ${ }^{13}$ for bleaching of vital teeth and recently it has been also recommended as an intracoronal bleaching ${ }^{24,28}$. Sodium perborate, which is frequently used in bleaching of non-vital teeth, decomposed in hydrogen peroxide after contact with water, releasing nascent oxygen ${ }^{29}$. Nowadays, the carbamide peroxide and the sodium perborate have been more used due to slight tissues damage caused by these agents.

In 1961, Spasser $^{27}$ recommended the use of sodium perborate and water inside the pulp chamber. In 1967, his technique was modified by Nutting and $\mathrm{Poe}^{20}$, who substituted the water by $30 \%$ hydrogen peroxide and suggested the term "walking bleach" be used to refer to the technique. These authors reported that sodium perborate and hydrogen peroxide release oxygen, so their combination should be synergistic and more effective. However, it has been discussing the effectiveness and damage of bleaching materials, especially the external cervical resorption ${ }^{18}$.

Friedman, et al. ${ }^{9}$ (1988) reported the incidence of external cervical resorption in $6.9 \%$ of the 58 non-vital teeth after intracoronal bleaching with sodium perborate and $30 \%$ hydrogen peroxide for a period of 1-8 years. However, Holmstrup, et al. ${ }^{14}$ (1988) did not verify external cervical resorption in pulpless teeth after intracoronal bleaching with sodium perborate mixed with water after evaluation of 3 years. Rotstein, et al. ${ }^{25}$ (1991) verified that sodium perborate with water presented similar effectiveness of bleaching compared to other bleaching materials, and prevented or minimized the occurrence of external root resorption. The efficacy of bleaching not only depends on the solvent used (water or hydrogen peroxide in different concentrations), but also depends on types of sodium perborate ${ }^{29}$.

Perborates differ from its content of oxygen which can be considered as a measure of its potential bleaching efficacy. Weiger, et al. ${ }^{29}$ (1994) compared the effects of various types of sodium perborate (mono, tri or tetrahydrate) used for intracoronal bleaching and verified that the combination of tetrahydrate sodium perborate with $30 \%$ hydrogen peroxide or water resulted in similar esthetic results. Ari and Üngör ${ }^{2}$ (2002) verified that no statistically significant difference in the esthetic results was found when the three types of sodium perborate were used with water or $30 \%$ hydrogen peroxide in intracoronal bleaching of pulpless teeth. Rotstein, et al. ${ }^{25}$ (1991) also showed that no statistically significant difference was observed between the sodium perborate mixed with water or hydrogen peroxide in intracoronal bleaching of discolored human teeth. However, Bizhang, et al. ${ }^{4}$ (2003) demonstrated in vitro that sodium perborate mixed hydrogen peroxide was more effective than sodium perborate mixed with water in intracoronal bleaching of discolored bovine teeth.

Evaluating bleaching materials with few damage effects,
Vachon, et al. ${ }^{28}$ (1998) compared the effectiveness of $10 \%$ carbamide peroxide and sodium perborate mixed with $30 \%$ hydrogen peroxide in internal bleaching of pulpless teeth, and verified that both presented similar bleaching efficacy. Perrine, et al. ${ }^{23}$ (2000) compared $10 \%$ carbamide peroxide to sodium perborate and water in the intracoronal bleaching and verified that, after 12 days of bleaching, $65 \%$ of the teeth were bleached with carbamide peroxide and $67 \%$ of the teeth were bleached with sodium perborate, with no statistical significance. Leonard, et al. ${ }^{15}$ (1998) compared the shade changes in extracted teeth during 2 weeks' whitening with $5 \%, 10 \%$ or $16 \%$ carbamide peroxide and verified that the higher concentrations (10\% and $16 \%)$ were more efficient. Lower concentrations of carbamide peroxide take longer to bleach teeth but eventually achieve the same result as higher concentrations. Yui et al..$^{30}$ (2008) showed that sodium perborate associated with both $10 \%$ and $35 \%$ carbamide peroxide was more effective than when associated with distilled water for intracoronal bleaching using walking bleaching technique. Other mixtures of sodium perborate have been evaluated, Oliveira, et al. ${ }^{21}$ (2006) verified that the combination of sodium perborate with $2 \%$ chlorhexidine gel did not decrease the efficacy of bleaching with sodium perborate and suggested that $2 \%$ chlorhexidine gel exhibited a good potential for use as a vehicle for sodium perborate or as a complement for carbamide peroxide during the walking bleaching technique to prevent coronal microleakage.

The efficacy and the necessary time for a good bleaching of different bleaching materials and mixtures have been evaluated, thus, it is interesting to evaluate if the mixture of carbamide peroxide and sodium perborate is more effective than these alone materials. Since there are few studies in the literature that evaluated the efficacy of $16 \%$ carbamide peroxide (intermediate concentration) for intracoronal bleaching using walking bleaching technique, the aim of this study was to evaluate the efficacy of $16 \%$ carbamide peroxide gel and tetrahydrate sodium perborate used as intracoronal bleaching of discolored teeth due to blood decomposition, after 7, 14 and 21 days exposure time.

\section{MATERIAL AND METHODS}

Sixty root-single human premolars with intact crowns were used. These teeth were freshly extracted from patients of the Dental School of São José dos Campos, São Paulo State University and the teeth was selected based on relative dimensions and similar morphology. Debris, calculus and soft tissue remnants on the root surfaces were cleaned using a Gracey curette and all teeth were stored in saline solution ( $\mathrm{NaCl} \mathrm{0.85 \% ,} \mathrm{Aster} \mathrm{Produtos} \mathrm{Médicos} \mathrm{Ltda,} \mathrm{Sorocaba,} \mathrm{SP,}$ Brazil) until used.

The coronal access opening was prepared using a diamond bur under high-speed water spray cooling. The root canal of each tooth was enlarged using a step-back technique followed by obturation with gutta-percha and root canal sealer (Sealapex, Kerr, Orange, USA) using lateral 
condensation. Two millimeters of root canal filling was removed below the vestibular cementoenamel junction and covered with a $1 \mathrm{~mm}$ thick cervical seal of resin-modified glass-ionomer cement ${ }^{22}$ (Vitremer, 3M, St. Paul, MN, USA).

Each tooth was identified and its original color was evaluated using Vita shade guide (VITA Zahnfabrik, Bad Säckingen, Germany). Standardized photographs of the vestibular face crown of all specimens were taken using Kodak Color film under standardized lighting conditions (original color). The photographic camera (Canon T50, automatic with ocular macro FD $100 \mathrm{~mm}, 1: 4$ and extension tube FD50; Canon, Japan) was positioned in a distance of $50 \mathrm{~cm}$ of the tooth. These films were developed under the same conditions by a professional laboratory. After, the teeth were stained with fresh red blood cells using the method described by Freccia and Peters ${ }^{8}$ (1982) and modified by Weiger, et al. ${ }^{29}$ (1994).

Each tooth was placed in an individual test tube containing $2 \mathrm{~mL}$ of fresh rabbit blood and then centrifuged at $500 \mathrm{Xg}$ for $30 \mathrm{~min}$, three times a day. The blood was replaced each day and the discoloration procedure repeated for 18 consecutive days, until similar discoloration was achieved in all specimens. The teeth were finally washed with distilled water and the crowns polished with a rubber cup and pumice. New standardized photographs of the buccal face of the crowns were taken and the color after artificial staining was evaluated using Vita shade guide.

The pulp chambers of all teeth received 17\% EDTA (Byoformula, São José dos Campos, SP, Brazil) for 3 min, followed by rinsing with saline solution. The teeth were randomly divided into four experimental groups $(n=15)$, according to the bleaching material used in the pulp chamber: Group 1 (CP): 16\% carbamide peroxide gel (Clarigel Gold, Dentsply, Petrópolis, RJ, Brazil); Group 2 (CP + SP): mixture of $16 \%$ carbamide peroxide gel with tetrahydrate sodium perborate (volume ratio $=1: 3$ ); Group $3(\mathrm{SP})$ : tetrahydrate sodium perborate (Byoformula) with distilled water in a ratio of $2 \mathrm{~g}$ of powder to $1 \mathrm{ml}$ of liquid; Group 4 (control): damp cotton pellet with distilled water.

After the pulp chambers filling with the bleaching materials, the access cavities were sealed with Cimpat (Septodont Brasil LTDA, SP, Brazil) and teeth were kept at $37 \pm 1^{\circ} \mathrm{C}$ in a humidified atmosphere. After 7 days, the color of the bleached teeth was evaluated. The temporary cement was removed and the bleaching material was washed out with water and a fresh portion of bleaching agent placed into the pulp chamber. This procedure was repeated twice in 7-day intervals.

The color of each specimen was evaluated using the Vita shade guide under standardized lighting conditions. Evaluation was performed at day $0,7,14$ and 21 by two endodontists working independently, who were previously calibrated in the pilot study. Only when there was no agreement between the two endodontists, was a tooth reevaluated by both to come to a common decision ${ }^{17}$.

Scores from 0 to 4 were assigned as follows: Score $0=$ no bleaching; Score 1 = bleaching of 1 degree in Vita shade guide, not returning to the original tooth color; Score $2=$ bleaching of 2 degrees in Vita shade guide, not returning to the original tooth color; Score 3 = bleaching of 3 or more degrees in Vita shade guide, not returning to the original tooth color; Score $4=$ satisfactory bleaching, returning the original tooth color.

For photographic analysis, all photos (original, immediately after artificial staining and after 7, 14 and 21 days of bleaching) of each specimen were available for 2 endodontists previously calibrated in a pilot study under standardized lighting conditions. The intracoronal bleaching efficacy was categorized from degrees 0 to 3 as follows (based on study of Ari and Üngor ${ }^{2}$ ): Degree 0: no bleaching of dental crown; Degree 1: unsatisfactory or slight bleaching; Degree 2: satisfactory bleaching, however, without reaching the original shade; Degree 3: satisfactory or complete bleaching of dental crown indicating that the discolored tooth had returned to its original shade.

The results obtained by Vita shade guide and by photographic examination were analyzed statistically using non-parametric Kruskal-Wallis and Dunn's tests. In all cases, $p$ values $<0.05$ were considered as statistically significant.

\section{RESULTS}

Analysis of Chrome Value Bleaching for the Vita Shade

Tables 1, 2 and 3 showed distribution of frequency absolute, relative and median of scores obtained using Vita shade guide after 7, 14 and 21 days of bleaching, respectively.

The experimental groups presented bleaching results statistically similar ( $>0.05$ ) at the end of 7, 14 and 21 days. These groups presented higher bleaching significantly than control group (G4) $(\mathrm{p}<0.05)$ (Tables 1,2 and 3). In the G1 group (CP), $80 \%$ of discolored teeth returned of original color or presented satisfactory bleaching at the end of 21 days. In the G3 group (SP), 93.33\% of discolored teeth returned of original color or presented satisfactory bleaching at the end of 21 days and in the $\mathrm{G} 2$ group $(\mathrm{CP}+\mathrm{SP}), 100 \%$ of discolored teeth returned of original color or presented satisfactory bleaching at the end of 21 days.

\section{Photographic Analysis}

The results of degrees obtained by photographic analysis after bleaching in different groups are showed in Table 4. Also, the distribution of frequency absolute, relative and median of degrees obtained at the end of 21 days can be observed in Table 4

There was no significant difference among the groups G1, G2 and G3 ( $>0.05)$ in the periods evaluated. The control group (G4) was statistically different from other groups $(p<0.05)$, presenting low or any degree of bleaching. Figures 1 to 4 showed bleaching obtained in evaluated groups at the end of 21 days. It was verified that $\mathrm{G} 2$ group promoted return of original color in $100 \%$ of teeth at the end of 21 days.

Both methods (Vita shade guide and photographic analysis) showed similar results, without statistical difference among them. 
TABLE 1- Distribution of absolute (n) and relative (\%) frequency and median of scores (Vita shade guide) obtained in the experimental groups 7 days post-bleaching

\begin{tabular}{|c|c|c|c|c|c|c|c|c|}
\hline \multirow[t]{2}{*}{ Scores } & \multicolumn{2}{|c|}{ G1 } & \multicolumn{2}{|c|}{ G2 } & \multicolumn{2}{|c|}{ G3 } & \multicolumn{2}{|c|}{ G4 } \\
\hline & $\mathbf{n}$ & $\%$ & $\mathbf{n}$ & $\%$ & $\mathbf{n}$ & $\%$ & $\mathbf{n}$ & $\%$ \\
\hline 0 & 0 & 0 & 0 & 0 & 1 & 0 & 15 & $100 \%$ \\
\hline 1 & 0 & 0 & 0 & 0 & 0 & 0 & 0 & 0 \\
\hline 2 & 0 & 0 & 0 & 0 & 0 & 0 & 0 & 0 \\
\hline 3 & 14 & $93.33 \%$ & 7 & $46.67 \%$ & 8 & $53.33 \%$ & 0 & 0 \\
\hline 4 & 1 & $6.67 \%$ & 8 & $53.33 \%$ & 6 & $46.67 \%$ & 0 & 0 \\
\hline median & \multicolumn{2}{|c|}{$3^{A}$} & \multicolumn{2}{|c|}{$4^{\mathrm{A}}$} & \multicolumn{2}{|c|}{$3^{A}$} & \multicolumn{2}{|c|}{$0^{B}$} \\
\hline
\end{tabular}

G1 - 16\% carbamide peroxide; G2 - 16\% carbamide peroxide + sodium perborate; G3 - sodium perborate + water; G4 control. *Medians followed by the same letter are not significantly different $(p>0.05)$.

TABLE 2- Distribution of absolute (n) and relative (\%) frequency and median of scores (Vita shade guide) obtained in experimental groups 14 days post-bleaching

\begin{tabular}{|c|c|c|c|c|c|c|c|c|}
\hline \multirow[t]{2}{*}{ Scores } & \multicolumn{2}{|c|}{ G1 } & \multicolumn{2}{|c|}{ G2 } & \multicolumn{2}{|c|}{ G3 } & \multicolumn{2}{|c|}{ G4 } \\
\hline & $n$ & $\%$ & $\mathbf{n}$ & $\%$ & $n$ & $\%$ & $\mathbf{n}$ & $\%$ \\
\hline 0 & 0 & 0 & 0 & 0 & 0 & 0 & 12 & $80 \%$ \\
\hline 1 & 0 & 0 & 0 & 0 & 0 & 0 & 0 & 0 \\
\hline 2 & 0 & 0 & 0 & 0 & 0 & 0 & 1 & $6.67 \%$ \\
\hline 3 & 6 & $40 \%$ & 4 & $26.67 \%$ & 1 & $6.67 \%$ & 2 & $13.33 \%$ \\
\hline 4 & 9 & $60 \%$ & 11 & $73.33 \%$ & 14 & $93.33 \%$ & 0 & 0 \\
\hline median & \multicolumn{2}{|c|}{$4^{A}$} & \multicolumn{2}{|c|}{$4^{A}$} & \multicolumn{2}{|c|}{$4^{A}$} & \multicolumn{2}{|c|}{$0^{B}$} \\
\hline
\end{tabular}

G1 - 16\% carbamide peroxide; G2 - 16\% carbamide peroxide + sodium perborate; G3 - sodium perborate + water; G4 control. *Medians followed by the same letter are not significantly different $(p>0.05)$.

TABLE 3 - Distribution of absolute (n) and relative (\%) frequency and median of scores (Vita shade guide) obtained in experimental groups 21 days post-bleaching

\begin{tabular}{|c|c|c|c|c|c|c|c|c|}
\hline \multirow[t]{2}{*}{ Scores } & \multicolumn{2}{|c|}{ G1 } & \multicolumn{2}{|c|}{ G2 } & \multicolumn{2}{|c|}{ G3 } & \multicolumn{2}{|c|}{ G4 } \\
\hline & $\mathbf{n}$ & $\%$ & $\mathbf{n}$ & $\%$ & $\mathbf{n}$ & $\%$ & $\mathbf{n}$ & $\%$ \\
\hline 0 & 0 & 0 & 0 & 0 & 0 & 0 & 12 & $80 \%$ \\
\hline 1 & 0 & 0 & 0 & 0 & 0 & 0 & 0 & 0 \\
\hline 2 & 0 & 0 & 0 & 0 & 0 & 0 & 1 & $6.67 \%$ \\
\hline 3 & 3 & $20 \%$ & 0 & 0 & 1 & $6.67 \%$ & 2 & $13.33 \%$ \\
\hline 4 & 12 & $80 \%$ & 15 & $100 \%$ & 14 & $93.33 \%$ & 0 & 0 \\
\hline median & \multicolumn{2}{|c|}{$4^{A}$} & \multicolumn{2}{|c|}{$4^{\mathrm{A}}$} & \multicolumn{2}{|c|}{$4^{A}$} & \multicolumn{2}{|c|}{$0^{B}$} \\
\hline
\end{tabular}

G1 - 16\% carbamide peroxide; G2 - 16\% carbamide peroxide + sodium perborate; G3 - sodium perborate+ water; G4 control. *Medians followed by the same letter are not significantly different $(p>0.05)$. 
TABLE 4- Distribution of of absolute (n) and relative (\%) frequency and median of degrees obtained in 4 groups by photographic analysis

\begin{tabular}{|c|c|c|c|c|c|c|c|c|}
\hline \multirow[t]{2}{*}{ Scores } & \multicolumn{2}{|c|}{ G1 } & \multicolumn{2}{|c|}{ G2 } & \multicolumn{2}{|c|}{ G3 } & \multicolumn{2}{|c|}{ G4 } \\
\hline & $\mathrm{n}$ & $\%$ & $\mathbf{n}$ & $\%$ & $\mathrm{n}$ & $\%$ & $\mathrm{n}$ & $\%$ \\
\hline 0 & 0 & 0 & 0 & 0 & 0 & 0 & 13 & $86.67 \%$ \\
\hline 1 & 0 & 0 & 0 & 0 & 0 & 0 & 2 & $13.33 \%$ \\
\hline 2 & 3 & $20 \%$ & 0 & 0 & 1 & $6.67 \%$ & 0 & 0 \\
\hline 3 & 12 & $80 \%$ & 15 & $100 \%$ & 14 & $93.33 \%$ & 0 & 0 \\
\hline median & \multicolumn{2}{|c|}{$3^{A}$} & \multicolumn{2}{|c|}{$3^{A}$} & \multicolumn{2}{|c|}{$3^{\mathrm{A}}$} & \multicolumn{2}{|c|}{0} \\
\hline
\end{tabular}

G1 - 16\% carbamide peroxide; G2 - 16\% carbamide peroxide + sodium perborate; G3 - sodium perborate+ water; G4 control. *Medians followed by the same letter are not significantly different $(p>0.05)$.
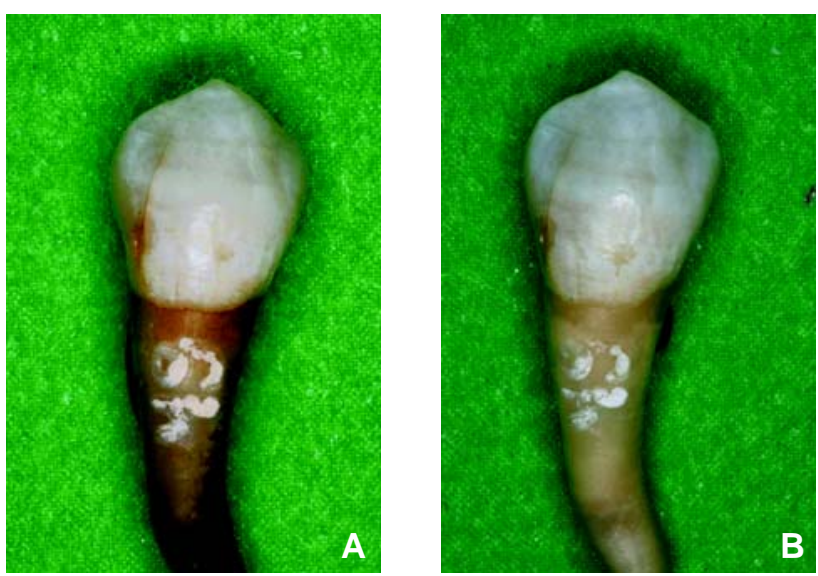

FIGURE 1- Specimen representative of control group (G4) after discoloration $(A)$ and after 21 days of bleaching $(B)$ with distilled water
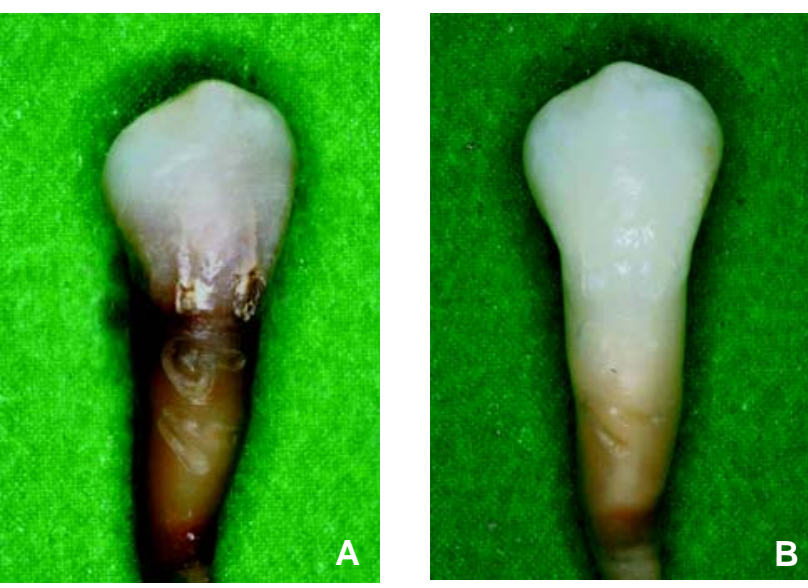

FIGURE 3- Specimen representative of group $\mathrm{G} 2$ after discoloration $(A)$ and after 21 days of bleaching $(B)$ with the mixture of $16 \%$ carbamide peroxide gel + tetrahydrate sodium perborate
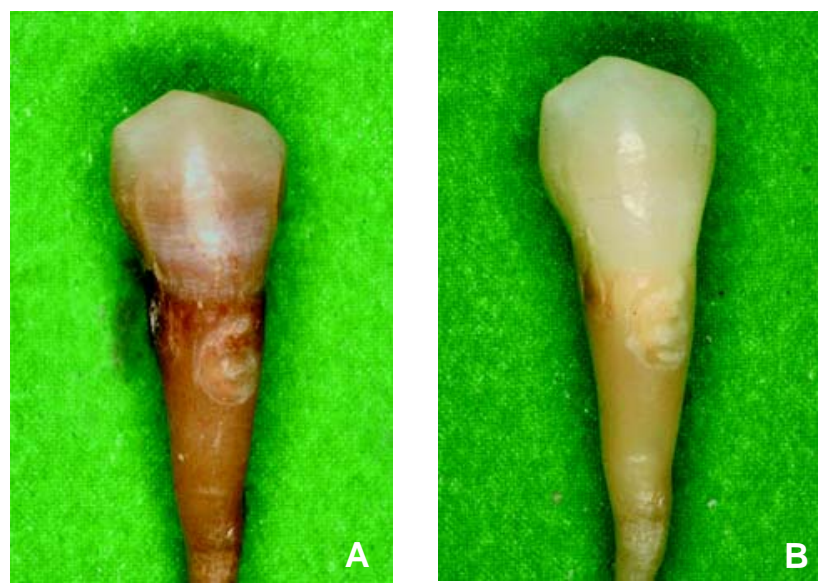

FIGURE 2- Specimen representative of group G1 after discoloration (A) and after 21 days of bleaching (B) with $16 \%$ carbamide peroxide gel
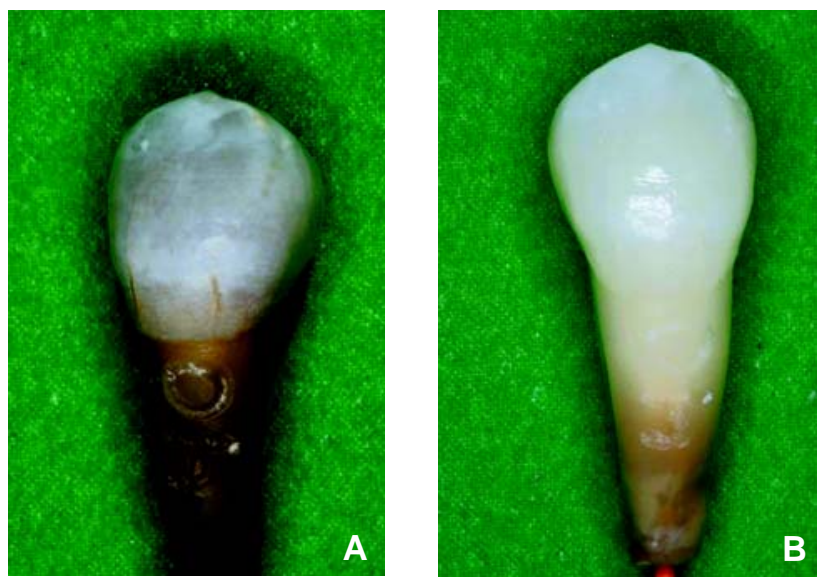

FIGURE 4- Specimen representative of group G3 after discoloration (A) and after 21 days of bleaching (B) with tetrahydrate sodium perborate 


\section{DISCUSSION}

In the present study, the teeth were discolored by blood decomposition for being an effective method to compare intracoronal bleaching materials ${ }^{8,21,25,29}$. The time of tooth discoloration recommend by Weiger, et al. ${ }^{29}$ (1994) was 10 days, but in the present study, this period was not sufficient for adequate discoloration, being performed by 18 consecutive days, according to Rotstein, et al. ${ }^{25}$ (1991) and Ari and Üngor ${ }^{2}$ (2002).

According to Lim, et al. ${ }^{17}$ (2004) it is well established that visual color determination is subjective, compared to the accuracy of spectrophotometer evaluation ${ }^{20}$. However, Vachon, et al. ${ }^{28}$ (1998) suggested that although the spectrophotometer readings might indicate a statistically significant difference, these differences could be clinically identical to the human eye. Therefore, some studies ${ }^{16,19}$ have used both spectrophotometer and visual to evaluate the effectiveness of bleaching agents in color change using the Vita Lumin shade guide and the results of these studies using both methods were consistent. In our study, the color changes in each specimen were evaluated using the Vita shade guide under standardized lighting conditions and by photographic analysis under standardized lighting conditions. According to the present results, both methods were efficient in the analysis showing no statistical difference among them. Thus, both methods (Vita shade guide and photographic analysis) can be recommended to evaluate the efficacy of bleaching agents applied in discolored teeth by blood decomposition.

Some studies ${ }^{1,10}$ have demonstrated that the mixture of sodium perborate with hydrogen peroxide presents excellent esthetic results, however, can cause root external cervical resorption. The cervical root resorption is one undesirable consequence of intracoronal bleaching ${ }^{17}$ and this has been attributed to excessive hydrogen peroxide diffusing into the periradicular tissues, possibly through cement defects ${ }^{17,25}$, although the exact mechanism has not been determined ${ }^{17}$. Ari and Üngor ${ }^{2}$ (2002) related that sodium perborate should be mixed with water rather than with hydrogen peroxide in order to prevent or minimize the occurrence of bleachingrelated external root resorption. Gökay, et al. ${ }^{11}$ (2008) evaluated and compared radicular peroxide diffusion from different concentrations of carbamide peroxide bleaching gels $(10,17$ and $37 \%)$ and a mixture of $30 \%$ hydrogen peroxide and sodium perborate. The results showed that higher peroxide penetration occurred with a mixture of $30 \%$ hydrogen peroxide and sodium perborate than with carbamide peroxide bleaching gels, and 37\% carbamide peroxide group also promoted greater peroxide penetration than the others carbamide peroxide groups $(\mathrm{p}<0.05)$. No statistically significant difference was found between $10 \%$ and $17 \%$ carbamide peroxide groups $(p>0.05)$. These authors concluded that peroxide penetration of carbamide peroxide gels was significantly lower than the mixture of hydrogen peroxide and sodium perborate and suggested that carbamide peroxide gels might promote less risk of postbleaching external root resorption. Therefore, further research should evaluate the efficacy of bleaching agents which promote less damage to dental and periodontal tissues.

In the present study, tetrahydrate sodium perborate and $16 \%$ carbamide peroxide were evaluated alone and mixed. Sodium perborate in the form of mono-, tri- or tetrahydrate is used as a $\mathrm{H}_{2} \mathrm{O}_{2}$-releasing agent. Weiger, et al. ${ }^{29}$ (1994) verified that monohydrate sodium perborate presents high amount of active oxygen (16\%), however, the mixture with water or hydrogen peroxide become it ineffective after one hour. The trihydrate sodium perborate presents $11.8 \%$ of active oxygen and setting within 1 day. However, although tetrahydrate sodium perborate presents small amount of active oxygen $(10.4 \%)$, its setting occurs after 7 days. Therefore, tetrahydrate sodium perborate is recommended because it remains active until the bleaching agent is changed (7 days). Additionally, the amount of hydrogen peroxide penetration depends on the form of sodium perborate ${ }^{29}$, and the penetration is significantly high in mixture of sodium perborate mono or tetrahydrate and hydrogen peroxide and less in mixture of trihydrate with hydrogen peroxide or mixture of tetrahydrate and water.

Weiger, et al. ${ }^{29}$ (2004) reported that the esthetic results of teeth bleached with a mixture of tetrahydrate sodium perborate and water or hydrogen peroxide were similar. Oliveira et al. ${ }^{21}$ (2006) also verified that the mixture of sodium perborate and hydrogen peroxide presented similar esthetic results compared to the combination with distilled water. In our study, tetrahydrate sodium perborate and water presented satisfactory esthetic results since after 21 days, $93.33 \%$ of teeth returned to the original color. These results are in agreement with several previous studies that demonstrated that the sodium perborate with water is an effective bleaching agent $t^{2,14,21,29}$.

In our study was used $16 \%$ carbamide peroxide gel because is an intermediate concentration among the bleaching agents more used $(10 \% \text { to } 37 \%)^{18}$. Carrasco, et al. ${ }^{6}(2003)$ reported that the $37 \%$ carbamide peroxide as an intracoronal bleaching increased dentinal permeability, which was not observed for $27 \%$ carbamide peroxide. In this way, the use of carbamide peroxide at lower concentrations should be recommended ${ }^{30}$. The degradation of $10 \%$ carbamide peroxide releases $6.4-7 \%$ of urea and 3 $3.6 \%$ of $\mathrm{H}_{2} \mathrm{O}_{2}$, and $35 \%$ carbamide peroxide releases 23 $25 \%$ of urea and $10-12 \%$ of $\mathrm{H}_{2} \mathrm{O}_{2}{ }^{12,13}$. According to Braun, et al. $^{5}$ (2007), the high concentration of bleaching agent promote faster tooth bleaching, however, considering the biological aspects, it is recommended to use the low concentration agent to avoid damage tissue. Perrine, et al. ${ }^{23}$ (2000) demonstrated effective bleaching in $65 \%$ of teeth using 10\% carbamide peroxide gel and Chng, et al. ${ }^{7}$ (2004) verified that carbamide peroxide, in high concentrations, was more effective than sodium perborate mixed with water. Lim, et al. ${ }^{17}$ (2004) verified that $35 \%$ carbamide peroxide and $35 \%$ hydrogen peroxide were effective for intracoronal bleaching, and significantly better than sodium perborate mixed with water after seven days. After 14 days, no significant differences were found among the groups. These authors concluded that $35 \%$ carbamide peroxide could be recommended as an effective alternative to hydrogen 
peroxide for intracoronal bleaching. However, in this concentration, the biological effects of carbamide peroxide to dental and periodontal tissues should be more studied. In present study, $16 \%$ carbamide peroxide gel promoted effective bleaching in $80 \%$ of teeth after 21 days, showing no significant differences in relation to other groups (sodium perborate mixed with water or $16 \%$ carbamide peroxide mixed with sodium perborate).

The mixture of tetrahydrate sodium perborate with $16 \%$ carbamide peroxide gel was effective in tooth bleaching, since after 21 days, $100 \%$ of teeth returned to original color or presented satisfactory bleaching. Furthermore, the mixture these bleaching agents were as effective as the $16 \%$ carbamide peroxide gel and the sodium perborate mixed with water. In contrast, Yui, et al. ${ }^{30}$ (2008) evaluated the effectiveness of three formulations of bleaching materials (sodium perborate + water; sodium perborate $+10 \%$ carbamide peroxide and sodium perborate $+35 \%$ carbamide peroxide) for intracoronal bleaching of root filled teeth using the walking bleach technique and the authors concluded that sodium perborate mixed with both 10 and $35 \%$ carbamide peroxide was more effective than when mixed with water. In our study, although the mixture of tetrahydrate sodium perborate with $16 \%$ carbamide peroxide gel promoted satisfactory bleaching in $100 \%$ of teeth, there was no statistically significant difference among the groups.

There are few studies in the literature that evaluated the efficacy of $16 \%$ carbamide peroxide in association or not with sodium perborate. Therefore, further investigations should be conducted, especially in vivo studies, since crown discoloration occurs due to pulpal hemorrhage after traumatic tooth injuries. Moreover, more studies should evaluate mixtures between different bleaching agents in order to obtain products with better bleaching outcomes, shorter treatment duration and less damage to dental tissues ${ }^{26}$.

\section{CONCLUSIONS}

The three bleaching agents $(16 \%$ carbamide peroxide gel, tetrahydrate sodium perborate + water and the mixture of $16 \%$ carbamide peroxide gel + tetrahydrate sodium perborate) were efficient in intracoronal bleaching of discolored teeth due to blood decomposition, especially after 21 days showing no statistical difference.

\section{ACKNOWLEDGEMENTS}

The authors gratefully acknowledge the financial support provided by FAPESP (Fundação de Amparo à Pesquisa do Estado de São Paulo).

\section{REFERENCES}

1- Amato M, Serena Scaravilli M, Farella M, Riccitiello F. Bleaching teeth treated endodontically: long-term evaluation of a case series. J Endod. 2006;32:376-8

2- Ari H, Üngör M. In vitro comparison of different types of sodium perborate used for intracoronal bleaching of discoloured teeth. Int Endod J. 2002;35:433-6

3- Assis BRP, Albuquerque RC. Bleaching of non-vital teeth for the technique walking bleach. Rev CROMG. 1999;5:31-7.

4- Bizhang M, Heiden A, Blunk U, Zimmer S, Seemann S, Roulet JS. Intracoronal bleaching of discolored non-vital teeth. Oper Dent. 2003;28:334-40.

5- Braun A, Jepsen S, Krause F. Spectrophotometric and visual evaluation of vital tooth bleaching employing different carbamide peroxide concentrations. Dent Mater. 2007;23(2):165-9.

6- Carrasco LD, Fröner IC, Corona SA, Pécora JD. Effect of internal bleaching agents on dentinal permeability of non-vital teeth: quantitative assessment. Dent Traumatol 2003;19(2):85-9.

7- Chng HK, Yap AU, Wattanapayungkul P, Sim CP. Effect of traditional and alternative intracoronal bleaching agents on microhardness of human dentine. J Oral Rehabil. 2004;31:811-6

8- Freccia WF, Peters DD. A technique for staining extracted teeth: a research and teaching aid for bleaching. J Endod. 1982;8:67-9.

9- Friedman S, Rotstein I, Libfeld H, Stabholz A, Heling I. Incidence of external root resorption and esthetic results in 58 bleached pulpless teeth. Endod Dent Traumatol. 1988;1:56-60.

10- Friedman S. Internal bleaching: long term outcomes and complications. J Am Dent Assoc. 1997;128:51-5.

11- Gökay O, Ziraman F, Çali Asal A, Saka OM. Radicular peroxide penetration from carbamide peroxide gels during intracoronal bleaching. Int Endod J. 2008;41:556-60.

12- Goldstein RE, Garber DA. Completes dental bleaching. Chicago: Quintessence, 1995; p.10-165.

13-Haywood VB, Heymann HO. Nightguard vital bleaching. Quintessence Int. 1989;20:173-6.

14- Holmstrup G, Palm AM, Lambjerg-Hansen H. Bleaching of discolored root-filled teeth. Endod Dent Traumatol. 1988;4:197-201.

15- Leonard RH, Sharma A, Haywood VB. Use of different concentrations of carbamide peroxide for bleaching teeth: an in vitro study. Quintessence Int. 1998;29(8):503-7.

16- Li Y, Lee SS, Cartwright SL, Wilson AC. Comparison of clinical efficacy and safety of three professional at-home tooth whitening systems. Compend Contin Educ Dent. 2003;24:357-60, 362, 364; quiz 378.

17- Lim MY, Lum SO, Poh RS, Lee GP, Lim KC. An in vitro comparison of the bleaching efficacy of $35 \%$ carbamide peroxide with established intracoronal bleaching agents. Int Endod J. 2004;37:483-8.

18- Montgomery S. External cervical resorption after bleaching the pulpless teeth. Oral Surg. 1984; 57:203-6.

19- Nathoo S, Santana E, Zhang YP, Lin N, Collins M, Klimpel K, et al. Comparative seven-day clinical evaluation of two tooth whitening products. Compend Contin Educ Dent 2001;22:599-604, 606; quiz 608.

20- Nutting EB, Poe GS. Chemical bleaching of discolored endodontically treated teeth. Dent Clin North Am, 1967;11:655-62. 
21- Oliveira DP, Gomes BPFA, Zaia AA, Souza-Filho FJ, Ferraz CCR. In vitro assessment of a gel base containing $2 \%$ chlorhexidine as a sodium perborate's vehicle for intracoronal bleaching of discolored teeth. J Endod, 2006;35:672-4

22- Oliveira LD, Carvalho CA, Hilgert E, Bondioli IR, Araujo MA, Valera MC. Sealing evaluation of the cervical base in intracoronal bleaching. Dent Traumatol. 2003;19:309-13.

23- Perrine GA, Reichl RB, Baisden MK, Hondrum SO. Comparison of $10 \%$ carbamide peroxide and sodium perborate goes intracoronal bleaching. Gen Dent. 2000;48:264-70.

24- Plotino G, Buono L, Grande NM, Pameijer CH, Somma F. Nonvital tooth bleaching: a review of the literature and clinical procedures. J Endod. 2008;34(4):394-407.

25- Rotstein I, Zalkind M, Mor C, Tarabeah A, Friedman S. In vitro efficacy of sodium perborate preparations used for intracoronal bleaching of discolored non-vital teeth. Endod Dent Traumatol. 1991;7:177-80.

26- Smith J, Montgomery S. Cervical leakage after internal bleaching procedures. J. Endod. 1992;18:476-81.

27- Spasser HF. A simple bleaching technique using sodium perborate. NY State Dent J. 1961;27:332-4.

28- Vachon C, Vanek P, Friedman S. Internal bleaching with $10 \%$ carbamide peroxide in vitro. Pract Period Aesthet Dent. 1998;10:1145-8.

29- Weiger R, Kuhn A, Löst C. Radicular penetration of hydrogen peroxide during intracoronal bleaching using various forms of sodium perborate. Int Endod J. 1994;27:313-7.

30- Yui KC, Rodrigues JR, Mancini MN, Balducci I, Gonçalves SE. Ex vivo evaluation of the effectiveness of bleaching agents on the shade alteration of blood-stained teeth. Int Endod J. 2008;41:485-92. 